\title{
PROBLEMS CONNECTED WITH CONVEXITY
}

\author{
Z.A. Melzak \\ (received April 7, 1965)
}

1. This article consists of a list of problems connected with the theory of convex sets in the Euclidean space $\mathrm{E}^{\mathrm{n}}$, although in some cases the connection is indirect and/or inessential. The assumptions may often be relaxed or otherwise changed, thus giving rise to a wide variety of questions.

One fairly general way of obtaining such problems is the following. The defining property of a convex set $A$ in $E^{n}$ is that if the points $x$ and $y$ are in $A$ then so is the straight segment $x y$; if $A$ is closed then it is convex if it contains the midpoint of any two of its points. We have now the following problem matrix: what are the sets $A$ which have a certain property $P$ (such as being closed, compact, finite, discrete, connected etc.) and are such that if $x$ and $y$ are any two points in $A$ then $a$ certain set $B(x, y)$ (e.g., the midpoint of the segment $x y$, a semicircle with the endpoints $x$ and $y$, a point equidistant from $x$ and $y$ etc.) is also in $A$ ? Here and later on it will be often tacitly assumed that the set $A$ in question has sufficiently many points to render the problem meaningful. As general references we mention the A.M.S. Symposium on Convexity, the recent books of Valentine on Convex Sets and of Hadwiger et al. on Combinatorial Geometry, and the book of Fejes-Toth on Packings and Coverings.

2. The problems of this section are of the nature of exercises and fairly simple solutions can be readily found for them.

(1) Let $\mathrm{T}$ be a non-degenerate triangle. Let $\mathrm{S}$ be a closed set, in the plane $P$ of $T$, with the property that if two of its points are vertices of a triangle similar to $T$ then the third vertex also belongs to $S$. Show that $S=P$.

Canad. Math. Bull. vol. 8, no. 5, October 1965. 
(2) A plane convex body has the property that any two of its points can be connected in it by a smooth convex arc which starts and ends at right angles to the straight segment connecting the two points. Show that the convex body is a set of constant width with a smooth boundary.

(3) Show that the closed circular disks are the only plane compact sets $K$ such that every two points of $K$ can be connected in $\mathrm{K}$ by a semicircle.

(4) Let $\mathrm{C}$ be a circle. If a rectangle has two opposite vertices inside $C$, then the other two vertices cannot both lie outside C. Show that the above property characterizes the circle uniquely in the class of plane Jordan curves.

(5) Show that if $P$ is a convex polygon with $\geq 5$ sides then there exists inside $P$ a point the sum of whose distances from the vertices of $P$ exceeds the circumference of $P$. Does the problem generalize to $n$ dimensions?

(6) According to an unproved conjecture of Heilbronn, some three out of any $n$ points in the unit square determine a triangle of area $<c_{1} n^{-2}$. Prove that if the $n$ points are vertices of a convex polygon then the conjecture holds with $c_{1} n^{-2}$ replaced by $c_{2} n^{-3}$.

(7) Let $K$ be a smooth convex body in $E^{n}$ and let $T$ be an $n$-dimensional simplex in arbitrary orientation relative to $K$. Show that a simplex similar and similarly oriented to $\mathrm{T}$ can be inscribed into $\mathrm{K}$.

(8) Let $P$ be a plane non-selfintersecting closed polygon, not necessarily convex, no three vertices of which are collinear. Let the vertices be enumerated in cyclic order: $v_{1}, v_{2}, \ldots, v_{n}$, going round $\mathrm{P}$ clockwise or counter-clockwise. Define a diagonal of order $k, 1 \leq k \leq[n / 2]$, to be any straight segment joining two vertices $v_{i}$ and $v_{j}$, $i<j$, such that either $j-i=k$ or $\mathrm{n}-(\mathrm{j}-\mathrm{i})=\mathrm{k}$. A diagonal is called internal if it lies in the interior of $\mathrm{P}$ (except for its endpoints). Show that for every integer $\mathrm{N}$ there are polygons such that no diagonal of order 
$<\mathrm{N}$ is internal. What is the minimum number of internal diagonals of a polygon with $\mathrm{n}$ vertices?

(9) Let $C$ be a smooth closed convex curve, which we imagine to be the boundary of an idealized billiard table. Let $\mathrm{x}$ be a point of $\mathrm{C}, \mathrm{a} k$-path from $\mathrm{x}$ is a trajectory of a (point) ball projected from $x$ into the interior of $C$ and reflecting according to standard law's, which is periodic and has exactly $\mathrm{k}$ distinct points in common with $\mathrm{C}$. Show that a $k$-path exists from every point of $C$, for any $k \geq 2$, if and only if $\mathrm{C}$ is of constant width.

3. In this section there are collected some problems, the answers to which, though unknown to the author, are probably not difficult to obtain.

(10) What are the plane closed sets $K$, such that for every two points $x$ and $y$ of $K$ there is in $K$ a third point at which the straight segment $x y$ subtends a fixed angle a ?

(11) What are the plane compact sets $K$ such that for every two points $x$ and $y$ of $K, K$ contains two sides of a right-angled triangle whose third side is the hypothenuse $x y$ ?

(12) What are the convex bodies in $E^{n}$, of diameter 1 and with smooth boundaries, such that with every two points they also contain every (smaller) circular arc joining the points, and having radius $>1$ ?

(13) Let $P$ be a convex polyhedron in $\mathrm{E}^{3}$ with volume $\mathrm{V}$ and sum of all edge-lengths $L$. Is it true that $V / L^{3} \leq 2^{-2} 3^{-11 / 2}$ and the equality holds if and only if $P$ is similar to a $\bar{r}$ ight prism whose base is an equilateral triangle with side-length equal to the height of the prism?

(14) Is it true that an $n$-dimensional equivalent of a regular octahedron (that is, a regular cross-polytope) can be inscribed into any convex body in $\mathrm{E}^{\mathrm{n}}$ ? - Into any smooth convex body in $\mathrm{E}^{\mathrm{n}}$ ?

(15) It is well known that an elephant may be placed inside the unit cube of sufficiently high dimension. This suggests the 
following: what is the smallest integer $n=n(k, d)$ such that every subset of $E^{k}$ of diameter $d$ is congruent to a subset of the $\mathrm{n}$-dimensional unit cube?

(16) Let $U$ be a compact convex set in $E^{n}$ in some given orientation relative to a Cartesian coordinate system whose origin is outside $U$. For $x=\left(x_{1}, \ldots, x_{n}\right) \in U$ let $f_{k}(x)=\left(\sum x_{i}^{k}\right)^{1 / k}, k=2,3, \ldots$, and let $u(k)$ be the unique point of $U$ for which $f_{k}(x)$ attains its minimum. Is it true that for every $j, \quad 1 \leq j \leq n$, the $j$-th coordinates of the points $u(2)$, $\mathrm{u}(3), \ldots$ form a monotone sequence?

(17) What is the smallest number of umbilic points on a smooth (class $\mathrm{C}^{3}$ ) surface in $\mathrm{E}^{3}$ of constant width?

(18) Let $B(r)$ denote the ball of radius $r$ about the origin in $\mathrm{E}^{\mathrm{n}}$. Let $\mathrm{K}$ be a compact convex set in $\mathrm{E}^{\mathrm{n}}$, contained in $B(R)$ and star-shaped from every point of $B(1)$. Let $F(x)$ be its distance function (this is taken with respect to the origin, and $x$ varies over the unit sphere about the origin). Then there is a constant $a$, such that $a+F(x)$ is the distance function of a convex body. What is the infimum of all such a, expressed as a function of $n$ and $R$ ?

(19) Let $K$ be a set of constant width 1 in $E^{n}$. Call a straight segment in $K$ of length 1 a diametral. What is the supremum of the diameter of the set of midpoints of all diametrals of $K$, taken over all such sets $K$ ?

(20) Let $C$ be a plane rectifiable Jordan curve of length 1 . C may be 'convexified' by the process of successive reflexions of its re-entrant parts in the support lines. The result is a closed convex curve of the same length. What other properties does it share with $C$ ? If $C$ is a polygon with $n$ sides, what is the largest number of reflexions which may be necessary before obtaining a convex polygon? Are there meaningful generalizations to $\mathrm{n}$ dimensions?

4. In the present section we collect some problems which approach what might be called research level. 
(21) A plane finite set $x_{n}$ consists of $n \geq 3$ points and contains together with any two points a third one, equidistant from them. Does $X_{n}$ exist for every $n$ ? Must it consist of points lying on some two concentric circles (one of which may reduce to a point)? How many distinct (that is, not similar) $\mathrm{X}_{\mathrm{n}}$ are there for a given $\mathrm{n}$ ? What happens if the finiteness condition is changed so that the set is required to be countable and discrete?

(22) What is the length of the shortest tree which connects the $n+1$ vertices of the regular simplex in $E^{n}$ of unit diameter?

(23) What are the necessary and sufficient conditions on two n-dimensional simplexes, in order that it be possible to move one of them into the interior of the other?

(24) The unit sphere $S$ in $E^{n}$ is to be approximated by the boundary of a convex n-dimensional polyhedron with $\mathrm{m}$ walls, $m \geq n+1$. The approximation is to be best possible in the Hausdorff set-metric. Take the $m$ outward-bound unit normals to the walls of the polyhedron and translate them so that they originate at the centre of the sphere $S$, thus obtaining $\mathrm{m}$ points in $\mathrm{S}$. Is it true that the approximation is best possible if the $m$ points are such that the minimum distance between them is largest possible?

(25) In $E^{n}$ let $A$ and $B$ be two concentric balls, $A$ of radius 1 and $B$ of radius $a, 0<a<1$. A compact set $K$ is contained in A and is star-shaped at every point of $B$. What is the least upper bound on the surface area (length, if $\mathrm{n}=2$ ) of the boundary of $\mathrm{K}$ ?

(26) Let $M$ be a smooth (class $C^{1}$ ) orientable twodimensional manifold without boundary, embedded in $E^{3}$. Let $M$ have the property that through every point of it there pass at least $n$ distinct circles lying entirely in $M$. Is it true that if $n=4$ then $M$ must be a sphere or a torus, and if $n=5$ then it must be a sphere? Is it true that if $n>1$ and $M$ is convex then it must be a sphere? Is it true that if $n>1$ and $M$ is simply connected then it must be a sphere? 
Similar problems could be set up with circles replaced by straight lines, and it seems that the process of inversion of $M$ in suitable centres might be useful in either case.

(27) Let $p_{i}$ and $q_{i}, i=1, \ldots, n$, be $2 n$ points in the plane $P$, such that for all $i \neq j, \quad\left|p_{i}-p_{j}\right|>\left|q_{i}-q_{j}\right|$. Is it possible to find, in $P, n$ smooth convex arcs $C_{i}(t)$, $0 \leq t \leq 1$, such that $C_{i}(0)=p_{i}, C_{i}(1)=q_{i}$ and every distance $\left|C_{i}(t)-C_{j}(t)\right|, i \neq j$, is a decreasing function of $t$ ? If not, is it possible after a suitable re-enumeration of the $q_{i}{ }^{\prime} s$ ?

(28) Let $L$ be the integer lattice in the plane. Can one characterize those convex polygons $P$ which have the property that a polygon similar to $P$ can be found, every side of which contains a lattice point?

(29) A point in the plane undergoes $n$ successive displacements of unit length, each displacement in random direction. What is the probability $\mathrm{p}(\mathrm{n})$ that the path of the point is a part of a closed convex polygon?

(30) Let $A$ be a sufficiently flat convex arc in the plane and let $B$ be any perfect nowhere dense subset of $A$. Then $B$ is a subset of an arc $C$ with the following property: every subarc of $C$ contains a subarc similar to $C$. Must $C$ have Hausdorff dimension $>1$ ? What is the intersection of $C$ with any straight line which cuts it in at least countably many points?

(31) Let $A$ and $B$ be convex bodies in $E^{n}$ and suppose that $B$ is in the interior of $A$. What are the necessary and sufficient conditions on $A$ and $B$, expressed perhaps in terms of the smoothness and/or singularities of their boundaries, in order that $A$ be the vector sum of $B$ and a convex body $C$ ?

(32) A function $f(z)$ of the complex variable $z$ has $n$ specified singularities on the unit circle and no others anywhere in the finite plane. Let $R_{m}, m \geq 0$, be the region to which $f$ can be continued by $\mathrm{m}$ successive power series continuations starting at the origin; $R_{0}$ is the origin, $R_{1}$ is the interior of 
the unit circle, $R_{2}$ is the union of the interiors of all circles whose centre is in $R_{1}$ and whose circumference (but not the interior) contains a singularity, and so on. How should the singularities be distributed so that the diameter of $R_{m}$ be smallest (largest) possible? What are the arcs composing the boundary of $\mathrm{R}_{\mathrm{m}}$ ? If $\mathrm{z}$ is a given complex number, what is the smallest $m$ such that $z$ is in the region $\mathrm{R}_{\mathrm{m}}$ ?

(33) Let $K$ be a convex body in $E^{n}$ symmetric with respect to the origin. Let the mean curvature $M$ exist at every point of the boundary of $K$, and let $M \geq c$. What is the infimum of the volume of bodies $K$, which satisfy the above conditions and contain a point of the integer lattice other than the origin?

(34) Let $\mathrm{H}$ be a cube in $\mathrm{E}^{\mathrm{n}}$ of edge-length $\mathrm{a}$, and let $\mathrm{k} n$-dimensional balls of unit radius be placed so that their centres are at random in $\mathrm{H}$. What is the probability that the union of the $k$ balls is a connected set? - That it has $s$ components? - That it has volume not exceeding $b$ times the volume of one ball?

(35) Are there, for $n \geq 3$, n-dimensional sets of constant width whose boundaries fail to be smooth in the following strong sense: there exists no (relative) open subset of the boundary at each point of which the body has a unique support plane?

(36) Let $N$ points be taken in $E^{n}$ subject only to the condition of general position (no two coincide, no three collinear, no four coplanar etc.). What is the smallest $n_{0}=n_{0}(n, k)$, such that if $\mathrm{N} \geq \mathrm{n}_{0}$ then the $\mathrm{N}$ points must contain $\mathrm{k}$ vertices of a convex n-dimensional polyhedron?

(37) Call a convex body $K$ in $E^{n}$ a half-body if part of its boundary lies in a plane $P$ and the union of $K$ and its reflexion in $P$ is convex. Let $L$ be a convex body in $E^{n}$ and let $H(L)$ denote the half-body (or one of them if there are more than one), of largest possible volume, which can be sliced off $L$ by a plane. What is the value of 


$$
c(n)=\inf _{L}\left[\operatorname{vol~H}(L) / \text { vol } L_{1}\right] ?
$$

(38) Let $K_{n}$ be a convex body in $E^{n}$. It is called a cylinder if it is (congruent to) a Cartesian product of a straight segment and some $K_{n-1}$. For a convex body $K$ in $E^{n}$, let $C(K)$ denote the cylinder of largest volume that can be inscribed into $\mathrm{K}$. By a process of double inscription (a cone into $\mathrm{K}$, a cylinder into the cone) one can show that

$$
\operatorname{vol} \mathrm{C}(\mathrm{K}) / \text { vol } \mathrm{K} \geq \mathrm{c}_{1} \mathrm{n}^{-1} \text {. }
$$

Can this be improved to

$$
\text { vol } C(K) / \text { vol } K \geq c_{2} n^{-a}, \quad a<1 ?
$$

(39) It is easy to show that the ball in $\mathrm{E}^{3}$ cannot be the convex hull of an arc of finite length. One may pose the following approximation problem. Let $K$ be a convex body in $E^{3}$ of diameter 1 and let $\varepsilon>0$ be given. What is the infimum $L(\varepsilon)$ of the length $L$ of an arc $C$ whose convex hull approximates $\mathrm{K}$ to within $\varepsilon$ (in the Hausdorff set-metric)? When $\mathrm{K}$ is the ball, E. Strauss shows (private communication) that

$$
L(\varepsilon)=\pi \varepsilon^{-1 / 2}+O\left(\varepsilon^{-1 / 6}\right) ;
$$

is it true that for any $\mathrm{K}$

$$
L(\varepsilon)=c(K) \varepsilon^{-1 / 2}+o\left(\varepsilon^{-1 / 2}\right) ?
$$

If so, what is the constant $c(K)$ in terms of integrals of functions of curvatures over the boundary of $\mathrm{K}$ ?

(40) Let $\mathrm{K}$ be a strictly convex body in $\mathrm{E}^{\mathrm{n}}$ of unit diameter and let $u$ be a direction; the equator $E(u)$ is the subset of the boundary of $K$, consisting of all points at which there exist support planes parallel to $u$. Every equator $E(\mathrm{u})$ is contained between a pair of parallel planes having points in common with it and orthogonal to $u$; let $d(K, u)$ be the distance between them. What is the value of

$$
f(n)=\sup _{K} \min _{u} d(K, u) ?
$$


(41) Let $f_{1}, \ldots, f_{n}$ be $n$ points in the plane, not necessarily all distinct, and let an n-ellipse with these $n$ points as foci be the locus of points for which the sum of the distances from the foci is constant. Then every $n$-ellipse is a convex curve (which may reduce to the empty set, a point or an interval). Is it true that every plane closed convex curve $C$ can be arbitrarily well approximated by an n-ellipse? Is this true under the further condition that the approximating n-ellipse Iie inside (outside) C ?

It is easy to show that with a suitable arrangement of pins and thread an n-ellipse with known foci, and passing through a given point, can be drawn in the same way as an ordinary ellipse. Suppose that we enlarge the classical Euclidean constructions, using ruler and compasses in the traditional sense, by allowing the construction of n-erlipses as described above. What constructions are now possible? Is it possible to trisect any angle? Is it possible to construct a segment whose length is a times the length of a given segment, for any positive algebraic a ?

(42) Let $C$ be a plane closed convex curve which can be arbitrarily well approximated by an inscribed polygon all of whose sides and diagonals have rational lengths. Must $\mathrm{C}$ be a circle?

(43) Does there exist in $E^{3}$ a smooth (class $C^{3}$ ) surface of constant width, other than the sphere, all of whose geodesics are closed?

University of British Columbia 